\title{
Probing vortex-core dynamics using current-induced resonant excitation of a trapped domain wall
}

\author{
RAI MORIYA ${ }^{1}$, LUC THOMAS ${ }^{1}$, MASAMITSU HAYASHI ${ }^{1}$, YAROSLAW B. BAZALIY ${ }^{2}$, CHARLES RETTNER ${ }^{1}$ \\ AND STUART S. P. PARKIN ${ }^{1 *}$
}

${ }^{1}$ IBM Almaden Research Center, San Jose, California 95120, USA

${ }^{2}$ University of South Carolina, Columbia, South Carolina 29208, USA

*e-mail: parkin@almaden.ibm.com

Magnetic domain walls in soft magnetic nanowires often exhibit a structure in which the magnetization curls within the plane of the nanowire around a singular point with out-of-plane magnetization, the vortex core ${ }^{1,2}$. Although the core is a small object, with a diameter of only $\sim 10 \mathrm{~nm}$ in permalloy ${ }^{2}$, its motion controls the dynamics of the entire wall, which can be several hundred nanometres in size. In particular, when a domain wall trapped at a pinning site is driven out of equilibrium by either a magnetic field or a spin-polarized current, the vortex core gyrates around its equilibrium position. The sense of gyration is determined by the polarity of the $\operatorname{core}^{3-7}$. Here, we show that spin-polarized a.c. currents can resonantly excite a vortex domain wall trapped at a notched site in a nanowire. The shape and magnitude of the resonance, measured from the nanowire's resistance, reveal both the elliptical trajectory of the vortex core as well as its polarity.

A magnetic vortex is characterized by two boolean parameters, the chirality and the core polarity. As shown schematically in Fig. 1a, the vortex chirality is defined by the direction of rotation of the magnetization around the vortex core, either clockwise or anticlockwise. The core polarity $p$ is defined by the core magnetization direction, either pointing out of, $p=+1$, or into, $p=-1$, the plane of the vortex. The magnetization dynamics of vortices within magnetic nanoelements, both discs and wires, strongly influences their response to both magnetic fields and to spin-polarized current. Whereas magnetic field interacts with magnetization through the Zeeman interaction, spin-polarized current interacts with magnetic moments through spin momentum transfer ${ }^{7}$. The response of vortices in magnetic discs to short field pulses $^{4}$ or radiofrequency fields ${ }^{8}$ has been explored using pumpprobe magnetic imaging techniques. In the latter case, it was shown that the polarity of the vortex core could be switched resonantly with an a.c. field. Similarly, it has been shown using magnetic force microscopy that a.c. spin-polarized currents can switch the polarity of a vortex core ${ }^{9}$.

Electrical detection of the resonant response of magnetic nanodevices to a.c. field ${ }^{10}$ and current ${ }^{11,12}$ has been demonstrated. This technique has been applied to probe the vortex-core dynamics both in magnetic discs ${ }^{13}$ and nanowires ${ }^{14}$. The current-induced dynamics of vortex domain walls confined in a nanowire are

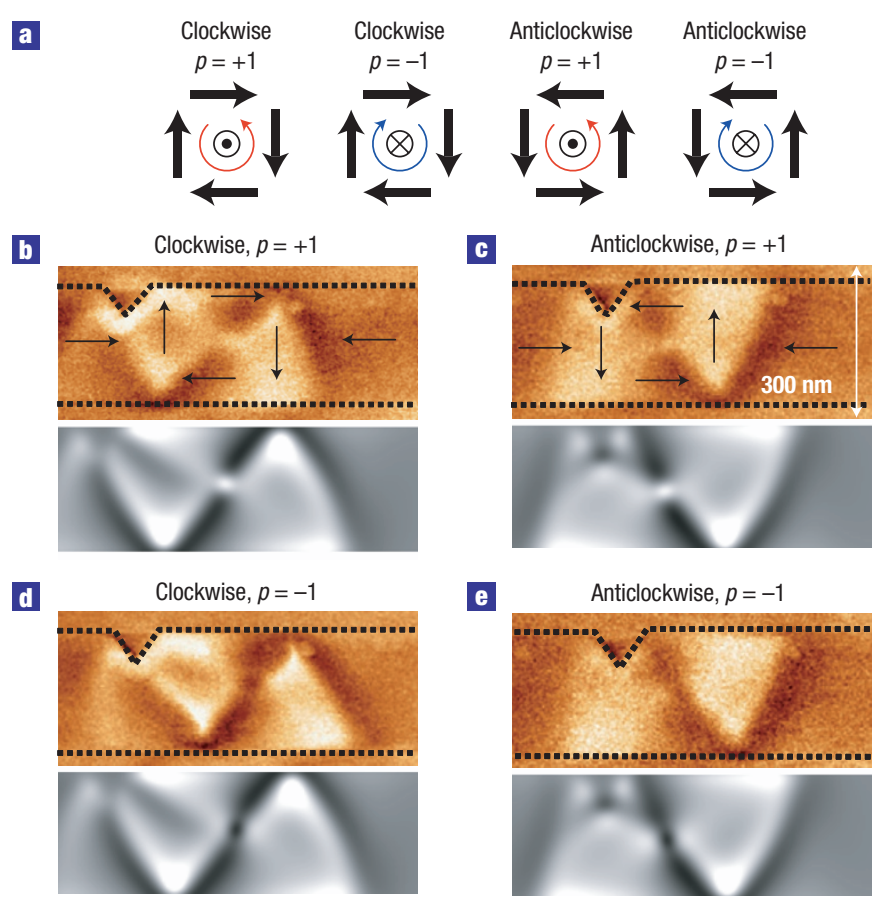

Figure 1 Magnetic images of four possible vortex domain wall structures. a, Schematic diagram of the magnetization profile of a vortex. Black arrows indicate the direction of magnetization. Two different chiralities, clockwise or anticlockwise, and core polarities, $p=+1$ or -1 , are shown. The direction of the gyrotropic rotation of the vortex core is shown as red (anticlockwise rotation) and blue (clockwise rotation) arrows. b-e, MFM images (top) and micromagnetic simulation of the MFM contrast (bottom) for all four different domain walls with clockwise $(\mathbf{b}, \mathbf{d})$ or anticlockwise (c,e) chiralities and core polarities $p=+1(\mathbf{b}, \mathbf{c})$ and $p=-1$ (d,e). Dotted lines show the shape of the triangular notch as measured by atomic force microscopy.

dominated by the motion of the vortex core ${ }^{15-18}$. For example, recent experiments ${ }^{19-21}$ have shown that the depinning of vortex 

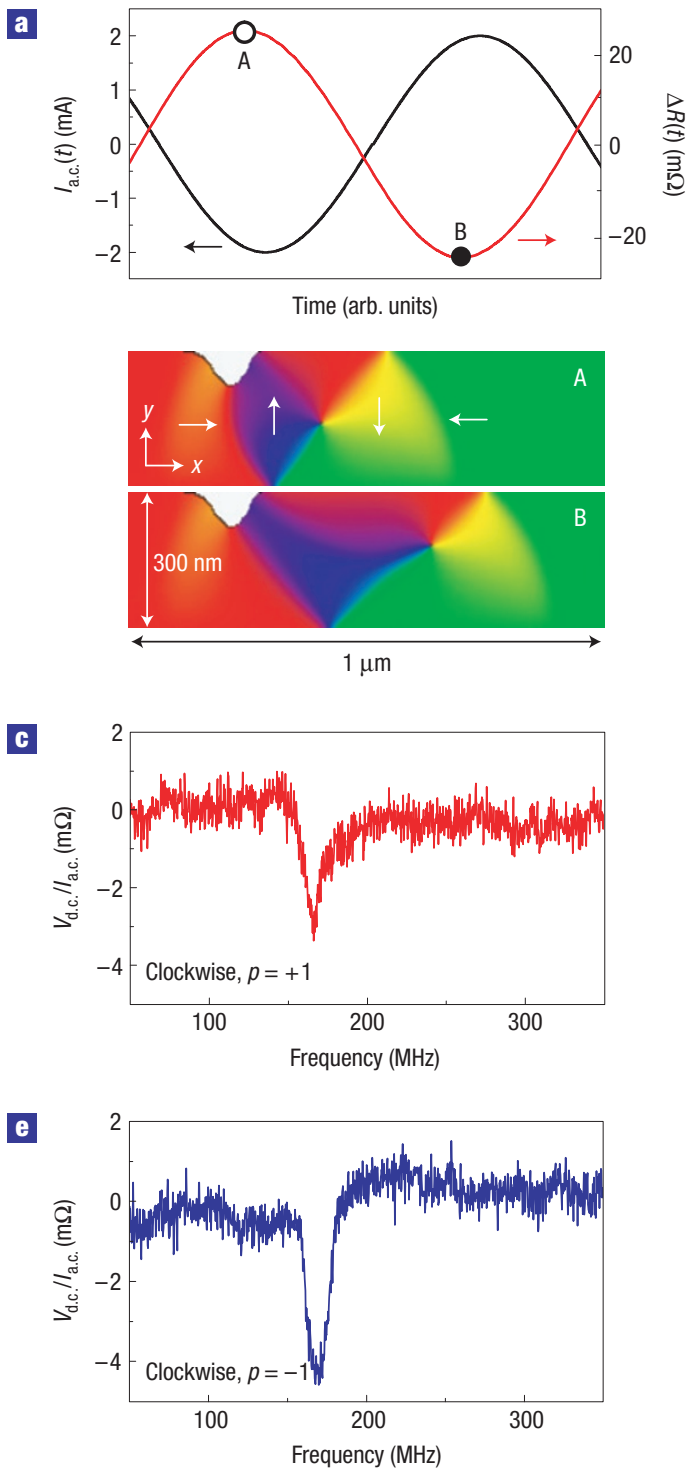

b
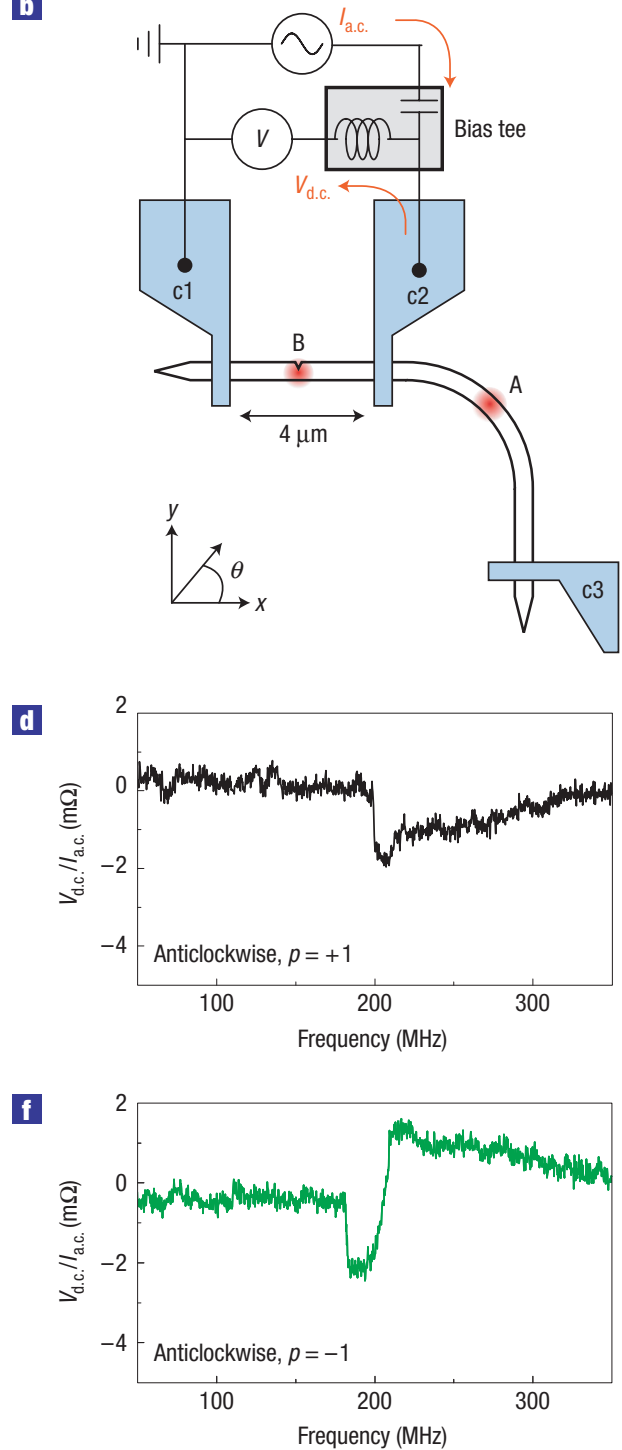

Figure 2 a.c.-current resonant excitation of vortex domain walls. a, Micromagnetic simulations of the resistance change under a.c.-current excitation for a clockwise domain wall. a.c.-current amplitude and frequency are $2.0 \mathrm{~mA}$ and $165 \mathrm{MHz}$, respectively. Snapshots of the domain wall structure at the resistance maximum (A) and minimum (B) are also shown. $\mathbf{b}$, Schematic diagram of the nanowire and the measurement circuit. Blue regions indicate the Rh/Ta contact. Positions $A$ and $B$ are defined in the Methods section. $\mathbf{c}-\mathbf{f}$, Frequency dependence of the d.c. voltage $V_{\text {d.c. }}$ measured for four different domain walls with clockwise $(\mathbf{c}, \mathbf{e})$ or anticlockwise $(\mathbf{d}, \mathbf{f})$ chiralities and core polarities $p=+1(\mathbf{c}, \mathbf{d})$ and $p=-1(\mathbf{e}, \mathbf{f})$. Measurements are carried out in zero magnetic field. The a.c.-current amplitude $l_{\text {a.c. }}$ is $0.52(\mathbf{c}, \mathbf{e})$ and $1.0 \mathrm{~mA}(\mathbf{d}, \mathbf{f})$. The measured voltage $V_{\text {d.c. }}$ is normalized by $l_{\text {a.c. }}$.

domain walls trapped at a local pinning potential by using current pulses or radiofrequency current is related to the gyrotropic motion of the vortex core ${ }^{22}$. Here, we show that the polarity of vortex domain walls pinned at a pinning site can be identified by the frequency dependence of their a.c.-current-driven resonant motion. Micromagnetic simulations and analytical modelling reveal that the shape of the resonance is correlated with the core rotation direction and thereby the polarity.

Experiments are carried out at room temperature on a permalloy $\left(\mathrm{Py}=\mathrm{Ni}_{81} \mathrm{Fe}_{19}\right)$ nanowire, $20 \mathrm{~nm}$ thick and $300 \mathrm{~nm}$ wide. Using a sequence of magnetic fields, a domain wall is injected and trapped at a notch in the nanowire that acts as a pinning site (see the Methods section). The injected domain wall exhibits one of four possible vortex structures (clockwise, anticlockwise with $p= \pm 1$ ) with approximately equal probability in successive injection events, as demonstrated by magnetic force microscopy (MFM). MFM images corresponding to each of these vortex structures are shown in Fig. 1b-e together with simulated magnetization patterns from micromagnetic modelling (see the Methods section and ref. 23). Very good agreement between the measured and simulated domain wall structures is found. The polarity of the core is clearly distinguishable by the bright (Fig. 1b,c) or dark spots (Fig. 1d,e) close to the centre of the vortex. Note that the core appears much larger in these images than in reality because the MFM is sensitive to the magnetic fields emanating from the sample and not directly to the magnetization ${ }^{1}$.

The presence of the domain wall results in a slight decrease of the nanowire's resistance arising from the anisotropic 
a

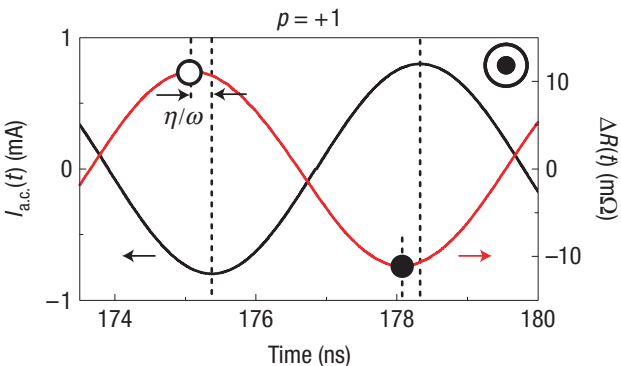

c

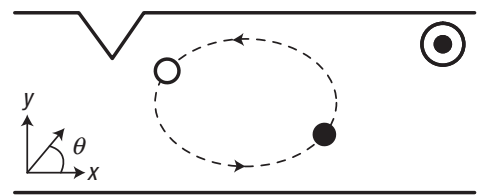

e

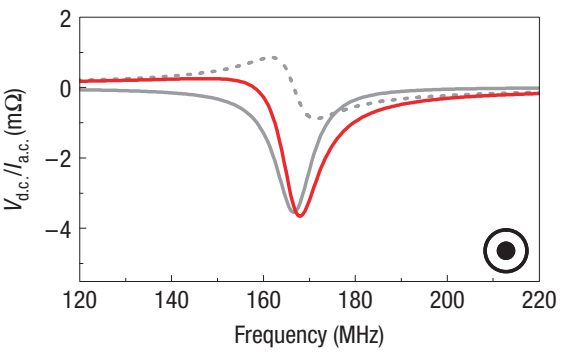

g

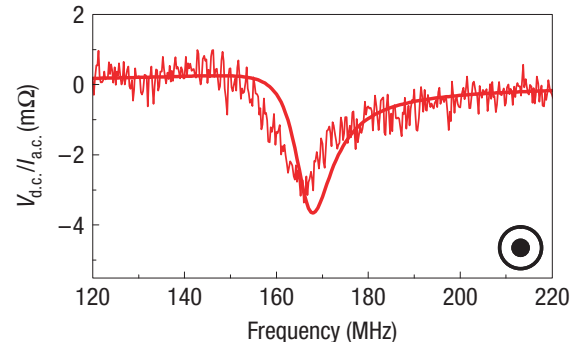

b

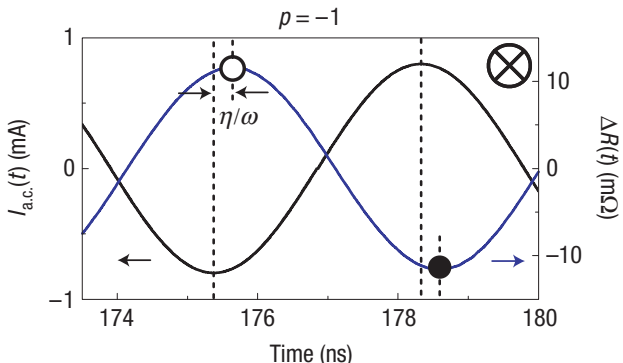

d

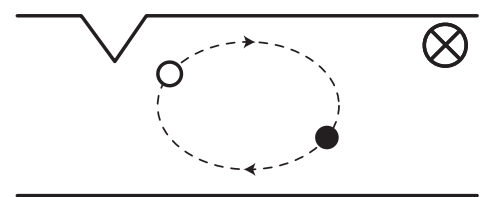

f

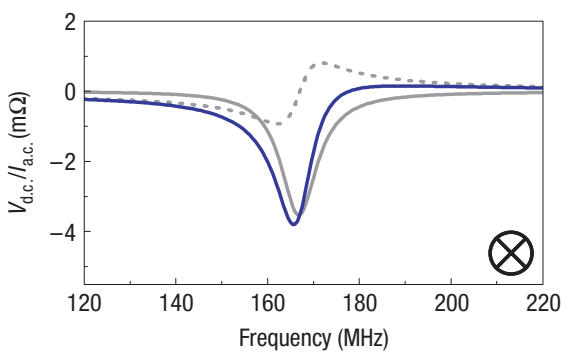

h

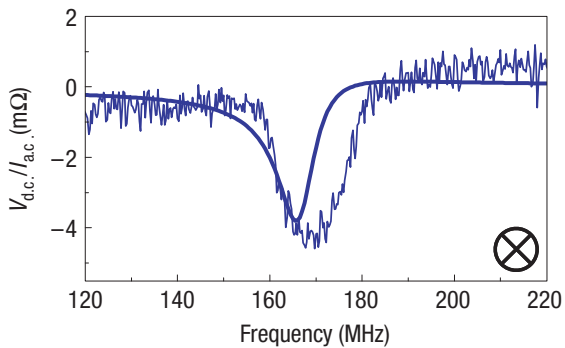

Figure 3 Polarity dependence of resonant peak shape and position. Left and right columns correspond to model and experimental results for clockwise domain walls with polarities of +1 and -1 , respectively. $\mathbf{a}, \mathbf{b}$, Micromagnetic simulations of the time dependence of the resistance during domain wall oscillations (red or blue lines) excited by an a.c. current (black line) with peak amplitude $0.8 \mathrm{~mA}$ and frequency $170 \mathrm{MHz}$. c,d, Schematic diagram showing the relationship between the domain wall resistance and the vortex-core position. Dotted lines and arrows show the trajectory and direction of the gyrotropic motion of the vortex core. When the core is located at the filled (open) black circle, the resistance is a minimum (maximum). e,f, Resonance peaks calculated from equation (1) with parameters (see text) to fit the experimental data shown in $\mathbf{g , h}$. Grey solid and dotted lines indicate the in-phase and out-of-phase components of $V_{\text {d.c. }}$ for $p= \pm 1$. Red and blue lines correspond to the sum of these in-phase and out-of-phase contributions. g,h, Comparison of the experimental data with the fitted curves for the resonance peaks measured at $l_{\text {a.c. }}=0.52 \mathrm{~mA}$.

magnetoresistance (AMR) of permalloy. The magnitude of the resistance change is proportional to the domain wall width ${ }^{24}$. Micromagnetic simulations show that when an a.c. current is passed across the domain wall at resonance, the resistance of the nanowire oscillates synchronously with that of the current with a small phase lag as shown in Fig. 2a. The resistance deviation from the equilibrium value $\Delta R(t)$ is calculated by assuming that it derives solely from $\mathrm{AMR}^{24,25} . \Delta R(t)$ results from a breathing mode of the domain wall which, because the domain wall is pinned at one end, contracts and expands as the current direction and the resulting spin torque is switched back and forth. The extrema magnetization distributions are shown in Fig. 2a.

As the device resistance $R(t)=R_{0}+\Delta R \cos (\omega t-\varphi)$ oscillates in response to the a.c. excitation current $I(t)=I_{\text {a.c. }} \cos (\omega t)$, a d.c. voltage $V_{\text {d.c. }}$ is generated across the nanowire due to the phase difference $\varphi$ between the current and resistance. $V_{\text {d.c. }}$ is given by $\left(I_{\text {a.c. }} \Delta R / 2\right) \cos \varphi$ (refs 11,12$)$ and is detected using the measurement circuit shown schematically in Fig. 2b. The measured frequency dependence of $V_{\text {d.c. }}$ (normalized by the a.c. current amplitude $I_{\text {a.c. }}$ ) is shown in Fig. $2 \mathrm{c}-\mathrm{f}$ for the four different vortex wall configurations pinned at the notch. Well-defined resonance peaks are observed in each case. Resonance frequencies of about 170 and $200 \mathrm{MHz}$ are measured for the clockwise (Fig. 2c,e) and anticlockwise (Fig. 2d,f) domain walls, respectively, showing a strong dependence on the domain wall's chirality but not on its polarity. This is because the resonance frequency is strongly influenced by the domain wall pinning potential, which itself depends on the detailed structure of the domain wall ${ }^{24}$ (see Supplementary Information, Fig. S1). The anticlockwise domain wall, which sits closer to the notch than the clockwise domain wall (Fig. 1), is consequently more strongly pinned at the notch and so exhibits a higher resonance frequency. The pinning potential 
is highly asymmetric because the domain walls sit to one side of the notch. The strength of the potential is reflected in the depinning fields, which we measure to be +46 and -180 Oe for the clockwise wall and +92 and -100 Oe for the anticlockwise wall, for depinning these walls to the right and to the left (see also ref. 24).

The influence of the vortex-core polarity $p$ is more subtle. As shown in Fig. $2 \mathrm{c}-\mathrm{f}$, the resonance peaks are asymmetric with respect to the resonance frequency. This asymmetry is different for opposite core polarities, but independent of the domain wall chirality. For vortices with positive polarities (Fig. 2c,d), the signal at the high-frequency side of the resonance peak is smaller than at the low-frequency side. In contrast, for vortices with negative polarities, the opposite behaviour is observed (Fig. 2e,f).

The distinctive features of the resonance peaks found experimentally for the four different vortex walls suggest that the a.c.-current-induced resonance can be used to probe not only the chirality of the wall but also the polarity of its core. Micromagnetic simulations show that this asymmetry results from a polarity-dependent phase shift between the a.c.-current and the AMR response of the domain wall. The time dependence of the resistance calculated under the influence of a.c. current for clockwise domain walls with core polarities of \pm 1 is shown in Fig. 3a,b. At the resonance frequency, $I(t)$ and $\Delta R(t)$ are nearly exactly out of phase. However, there is a small frequencyindependent phase offset $\eta$. This phase offset is related to the interplay between the two-dimensional trajectory of the vortex core and the symmetry-breaking notch as shown schematically in Fig. 3c,d. At resonance, the vortex core follows an elliptical trajectory around its equilibrium position moving to and fro both along the length and across the width of the nanowire. However, owing to the asymmetric notch, the maximal and minimal values of the domain wall's resistance do not exactly coincide with the outermost and innermost extent of the domain wall's trajectory along the length of the nanowire. This is the primary cause of the phase offset $\eta$ (see the Supplementary Information). Moreover, because the rotation direction is opposite for the two core polarities (anticlockwise and clockwise for $p=+1$ and -1 , respectively), $\eta$ also changes sign depending on the core polarity.

An analytical model can be used to calculate the resistance change due to the vortex-core motion. It follows that $V_{\text {d.c. }}$ can be expressed as (see the Supplementary Information):

$$
\frac{V_{\text {d.c. }}}{I_{\text {a.c. }}}=\frac{C}{2}\left\{X^{\prime} \cos \eta+p Y^{\prime} \sin \eta\right\},
$$

where $C$ is a constant that relates changes in the resistance with the changes in the vortex-core position for small perturbations of the core, $\eta$ is the phase difference between the resistance oscillation and core gyration and $\left(X^{\prime}, Y^{\prime}\right)$ are the amplitudes of the core gyration in the $x, y$ plane. Fits to the experimental data using equation (1) are shown as red and blue lines in Fig. $3 g$,h, for clockwise domain walls with $p= \pm 1$. Best fits are obtained with the parameters: $\alpha=0.009, \beta=2 \alpha$ and $\eta=0.22 \pi$, where $\alpha$ and $\beta$ are the Gilbert damping and the non-adiabatic contribution to the spin-transfer torque, respectively ${ }^{17}$. Note that the value of the phase shift $\eta$ is in excellent agreement with that calculated using a geometrical model of a pinned domain wall to calculate the resistance oscillation, as described in Supplementary Information.

The two terms in equation (1) correspond to dissipation and fluctuation-like terms, as shown by the grey solid and dotted lines in Fig. 3e,f. The first term is independent of the core polarity, whereas the sign of the second term depends on the core polarity. As a consequence of the superposition of these two components, the resonance peak exhibits an asymmetric structure (red and blue lines in Fig. 3e,f) that depends on the core polarity. The asymmetry of the experimental data is well reproduced.
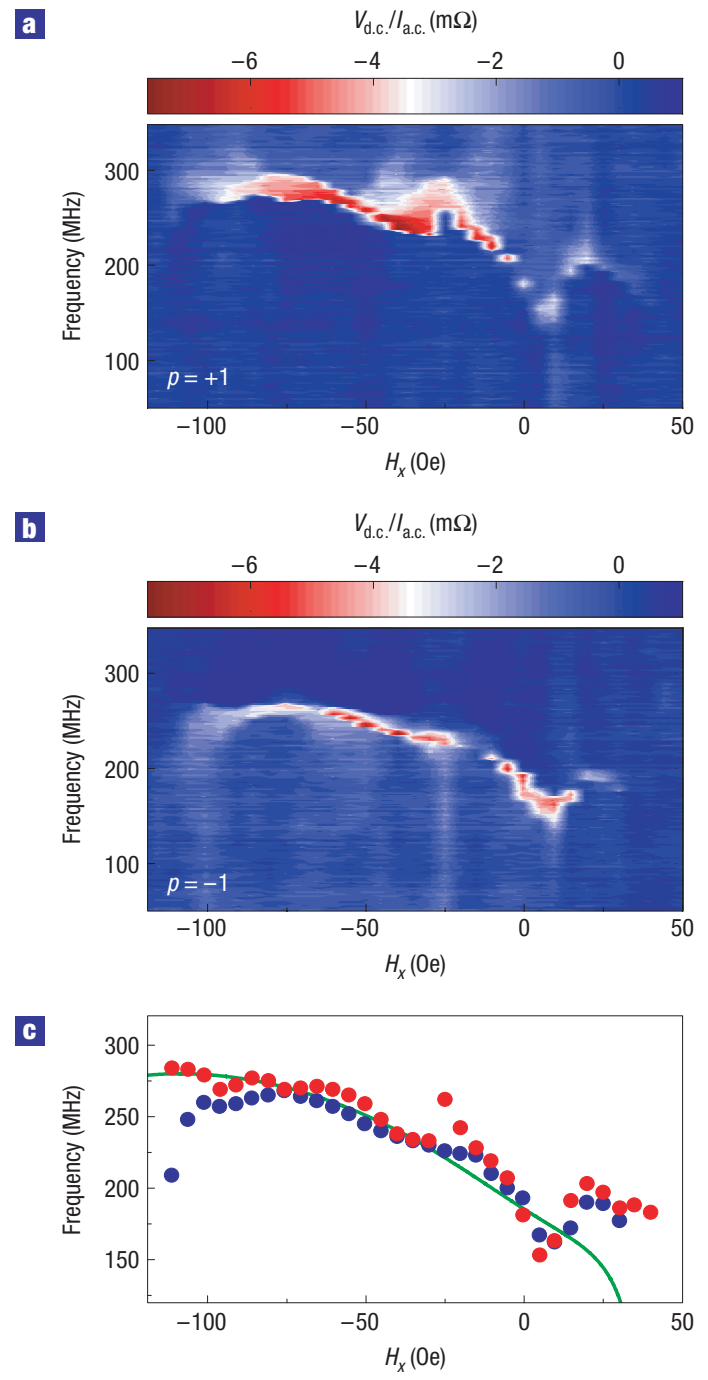

Figure 4 Determination of domain wall pinning potential from field dependence of resonance peak position. $\mathbf{a}, \mathbf{b}$, Contour plots of the magnetic field dependence of the clockwise domain wall resonance spectra with core polarities of $p=+1$ and -1 . The magnetic field is applied along the length of the nanowire. The a.c.-current amplitude $I_{\text {a.c. }}$ is $0.65 \mathrm{~mA}$. c, Measured resonance peak position versus external magnetic field $H_{x}$ for $p=+1$ (red circles) and $p=-1$ (blue circles) and comparison with model (green line).

We now show that the a.c.-current-induced resonance can be used to directly infer the pinning potential of the domain wall by measuring the dependence of the resonant frequency on the applied field. When a static external magnetic field $H_{x}$ is applied, which is below the depinning field, the domain wall shifts its equilibrium position towards or away from the notch, depending on the sign of $H_{x}$. As the pinning potential is non-parabolic, the pinning strength varies with the position of the domain wall. Figure $4 a, b$ compares the field dependence of the resonance spectra for clockwise domain walls with positive and negative core polarities. The resonance frequency strongly depends on the magnetic field, but is little influenced by the core polarity. This is because the field-dependent equilibrium position of the domain wall does not depend on the core polarity.

The domain wall resonance frequency, estimated by calculating the internal energy of the domain wall using micromagnetic 
simulations ${ }^{24}$, is compared with the experimental values in Fig. 4c. The good agreement shows that a measurement of the a.c.-current-induced resonance excitation frequency as a function of magnetic field can be used to experimentally probe the potential in which a domain wall is trapped. The magnetic field is used to move the equilibrium position of the domain wall in the potential well and so probe the spatial variation of the local pinning strength (which is proportional to the local curvature of the pinning potential). Note that in Fig. 4a,b, the resonance peak intensities decrease strongly for fields larger than +30 and -110 Oe, irrespective of the domain wall's polarity. This is because the domain walls depin from the notch above these fields, even though these fields are smaller than the zero-current depinning fields. The domain wall's motion is resonantly amplified by the a.c. current, which thereby assists depinning of the domain wall at lower magnetic fields ${ }^{19-21}$.

\section{METHODS}

\section{SAMPLE PREPARATION AND MEASUREMENT SET-UP}

The permalloy nanowire is formed from a film composed of 100 $\mathrm{Al}_{2} \mathrm{O}_{3} / 200 \mathrm{Ni}_{81} \mathrm{Fe}_{19} / 10 \mathrm{TaN} / 50 \mathrm{Ru}$ (units in $\AA$ ), which was deposited by magnetron sputtering on a thermally oxidized $\mathrm{Si}(100)$ substrate. The nanowire is patterned using electron beam lithography and argon ion milling. Its edges are then sealed by ion-beam-sputter-deposited alumina. Electrical contacts, made from $50 \mathrm{Ta} / 450 \mathrm{Rh}$ (units in $\AA$ ), are then fabricated in a lift-off process. Before deposition of the contacts, the surface of the nanowire is cleaned in situ by argon ion milling to promote a low contact resistance. A triangularly shaped notch is patterned on one side of the nanowire with dimensions of $\sim 80 \mathrm{~nm} \times 150 \mathrm{~nm}$

Electrical connections to the device are made using 7-GHz-bandwidth radiofrequency contact probes. Both the a.c.-current excitation at a frequency $\omega, I(t)=I_{\text {a.c. }} \cos (\omega t)$ and the output d.c. voltage $V_{\text {d.c. }}$ across the sample are measured using the same probe through a bias tee. Note that the data in Fig. 2 correspond to a higher value of $I_{\text {a.c. }}$ for the anticlockwise walls $(1.0 \mathrm{~mA})$ than for the clockwise walls $(0.52 \mathrm{~mA})$.

\section{DOMAIN WALL INJECTION PROCEDURE}

A large magnetic field (400 Oe) is first applied at an angle $\theta=45^{\circ}$ to the nanowire (Fig. 2b). After the field is reduced to zero, a head-to-head domain wall is created at position A in Fig. 2b. A magnetic field $H_{x} \sim-80$ Oe is then applied parallel to the left leg of the nanowire to move the domain wall from position $\mathrm{A}$ to position $\mathrm{B}$, where the domain wall is pinned at the triangularly shaped notch at one side of the wire. The d.c. resistance of the nanowire between the two contacts $\mathrm{c} 1$ and $\mathrm{c} 2$ is measured in zero magnetic field when the domain wall is outside these contacts at point $\mathrm{A}\left(R_{\mathrm{A}}\right)$ and when it is pinned at the notch $\left(R_{\mathrm{B}}\right)$. The domain wall resistance, $R_{\mathrm{DW}}=R_{\mathrm{B}}-R_{\mathrm{A}}$, depends on the domain wall structure (vortex or transverse) and its chirality (clockwise or anticlockwise) ${ }^{24}$. Three different values of $R_{\mathrm{DW}}$ are found: $-0.184,-0.176$ and $-0.132 \Omega$, corresponding to anticlockwise, clockwise vortex walls and a clockwise-transverse wall, as confirmed by MFM. Note that all domain walls are located at the right side of the notch because they are injected from the right part of the nanowire.

\section{MICROMAGNETIC SIMULATIONS}

Micromagnetic simulations are carried out on Py nanowires with the same physical dimensions as those used in the experiments ( $300 \mathrm{~nm}$ wide, $20 \mathrm{~nm}$ thick). We used typical material parameters for Py: exchange stiffness constant $A=1.3 \mu \mathrm{erg} \mathrm{cm}^{-1}$, zero magnetocrystalline anisotropy, Gilbert damping $\alpha=0.01$, non-adiabatic contribution to the spin-transfer torque $\beta=0$, spin polarization $P=0.4$ and saturation magnetization $M_{\mathrm{s}}=800$ e.m.u. $\mathrm{cm}^{-3}$. The finite-element cell size used is $5 \times 5 \times 20 \mathrm{~nm}^{3}$.

\section{Received 25 December 2007; accepted 10 March 2008; published 13 April 2008.}

\section{References}

1. Shinjo, T., Okuno, T., Hassdorf, R., Shigeto, K. \& Ono, T. Magnetic vortex core observation in circular dots of permalloy. Science 289, 930-932 (2000).

2. Wachowiak, A. et al. Direct observation of internal spin structure of magnetic vortex cores. Science 298, 577-580 (2002).

3. Guslienko, K. Y. et al. Eigenfrequencies of vortex state excitations in magnetic submicron-size disks. J. Appl. Phys. 91, 8037-8039 (2002).

4. Choe, S. B. et al. Vortex core-driven magnetization dynamics. Science 304, 420-422 (2004).

5. Buchanan, K. S. et al. Soliton-pair dynamics in patterned ferromagnetic ellipses. Nature Phys. 1, $172-176$ (2005).

6. Novosad, V. et al. Magnetic vortex resonance in patterned ferromagnetic dots. Phys. Rev. B 72, 024455 (2005).

7. Shibata, J., Nakatani, Y., Tatara, G., Kohno, H. \& Otani, Y. Current-induced magnetic vortex motion by spin-transfer torque. Phys. Rev. B 73, 020403 (2006)

8. Van Waeyenberge, B. et al. Magnetic vortex core reversal by excitation with short bursts of an alternating field. Nature 444, 461-464 (2006).

9. Yamada, K. et al. Electrical switching of the vortex core in a magnetic disk. Nature Mater. 6 270-273 (2007)

10. Grollier, J., Costache, M. V., van der Wal, C. H. \& van Wees, B. J. Microwave spectroscopy on magnetization reversal dynamics of nanomagnets with electronic detection. J. Appl. Phys. 100 024316 (2006).

11. Sankey, J. C. et al. Spin-transfer-driven ferromagnetic resonance of individual nanomagnets. Phys. Rev. Lett. 96, 227601 (2006)

12. Tulapurkar, A. A. et al. Spin-torque diode effect in magnetic tunnel junctions. Nature $\mathbf{4 3 8}$, 339-342 (2005).

13. Kasai, S., Nakatani, Y., Kobayashi, K., Kohno, H. \& Ono, T. Current-driven resonant excitation of magnetic vortices. Phys. Rev. Lett. 97, 107204 (2006).

14. Bedau, D. et al. Detection of current-induced resonance of geometrically confined domain walls. Phys. Rev. Lett. 99, 146601 (2007).

15. Berger, L. Possible existence of a Josephson effect in ferromagnets. Phys. Rev. B 33, 1572-1578 (1986).

16. Berger, $L$. Exchange interaction between electric current and magnetic domain wall containing Bloch lines. J. Appl. Phys. 63, 1663-1669 (1988).

17. Thiaville, A., Nakatani, Y., Miltat, J. \& Suzuki, Y. Micromagnetic understanding of current-driven domain wall motion in patterned nanowires. Europhys. Lett. 69, 990-996 (2005).

18. He, J., Li, Z. \& Zhang, S. Current-driven vortex domain wall dynamics by micromagnetic simulations. Phys. Rev. B 73, 184408 (2006).

19. Thomas, L. et al. Oscillatory dependence of current-driven magnetic domain wall motion on current pulse length. Nature 443, 197-200 (2006).

20. Thomas, L. et al. Resonant amplification of magnetic domain-wall motion by a train of current pulses. Science 315, 1553-1556 (2007).

21. Nozaki, T. et al. Substantial reduction in the depinning field of vortex domain walls triggered by spin-transfer induced resonance. Appl. Phys. Lett. 91, 082502 (2007).

22. Hung, C. Y., Berger, L. \& Shih, C. Y. Observation of a current-induced force on Bloch lines in Ni-Fe thin films. J. Appl. Phys. 67, 5941-5943 (1990).

23. LLG Micromagnetic Simulator < http://llgmicro.home.mindspring.com> (1997).

24. Hayashi, M. et al. Dependence of current and field driven depinning of domain walls on their structure and chirality in permalloy nanowires. Phys. Rev. Lett. 97, 207205 (2006).

25. Kent, A. D., Yu, J., Rüdiger, U. \& Parkin, S. S. P. Domain wall resistivity in epitaxial thin film microstructures. J. Phys. Condens. Matter 13, R461-R488 (2001).

Supplementary Information accompanies this paper on www.nature.com/naturephysics.

\section{Acknowledgements}

We thank X. Jiang for helpful discussions and sample fabrication and B. Hughes and K. Roche for technical assistance.

\section{Author information}

Reprints and permission information is available online at http://npg.nature.com/reprintsandpermissions Correspondence and requests for materials should be addressed to S.S.P.P. 